\title{
38
}

\section{Two Heuristics for Multicasting in ATM Networks}

\author{
F. Bernabei, A. Coppi, R. Winkler \\ Fondazione Ugo Bordoni \\ Via B. Castiglione, 59 - 00142 Roma Italy \\ Fax: +39654804404; e-mail: \{bernabei, wnk\}@fub.it
}

\begin{abstract}
This paper deals with multicasting in ATM networks. An overlaid network is proposed, that includes the ATM switches that provide the required signaling and information replication capabilities. These switches are interconnected by means of point-point Virtual Paths and run a hop-limited point-point routing scheme to configure the point-multipoint connections. The hop-limit constraint is embedded in the two considered multicasting algorithms, designed for operation in directed graphs. One algorithm modifies the well known KMB heuristic, originally developed for undirected graphs and now able to operate on directed graphs with the hop limit constraint. The other one modifies the Dijkstra algorithm, by adopting a cost function that considers the link capacity instead of its length. The performance of the two heuristics are quite close under many different operating conditions, but the modified Dijkstra has a lower algorithmic complexity.
\end{abstract}

Keywords

Multicast algorithms, hop limited routing, ATM, directed graphs

\section{INTRODUCTION}

The availability of efficient point-multipoint information transport facilities is one of the key issues for multiparty communications in ATM networks. The term "multicasting" has entered in the common vocabulary to refer to the transport of point-multipoint information using a single user to network transaction (in datagram networks) or a single connection (in virtual channel oriented networks).

This paper proposes an architecture and compares two heuristics for multicasting in ATM networks.

The architecture consists of an overlaid logical network, the Multicast Logical Network (MLN), composed of those ATM switches that provide the capabilities perform multicasting, i.e. the replication of user information and the in-call control of point-multipoint connections. These Multicast Capable Switches (MCS's) are connected by means of Virtual Paths (VP's), that traverse transparently the ATM switches that do not replicate information flows and are 
unable to control point-multipoint connections. The MCS's cooperate to establish, release and modify the point-multipoint connections, following the users' requests and according to the stream characteristics and the status of the MLN. Each user connects to one MCS, under the control of its Local Exchange. Thus, a point-multipoint connection is implemented by means of a two-tier architecture: one tier is the actual point-multipoint connection inside the MLN, the other tier is the set of point-point connections between the communicating parties and the relevant MCS's.

To route a point-multipoint connection the MCS's run a real time point-point routing scheme and a multicasting heuristic. The point-point routing scheme may be derived from the those currently adopted for evolving POTS and SS7 networks [Ash, 1994]. These mechanisms limit the maximum number of hops between source and destination, an effective way to simplify the processing and to reduce the number of switches to be traversed.

The multicasting heuristic uses the information provided by the routing scheme about the existing and suitable point-point routes, to establish a point-multipoint connection rooted at the MCS relevant to the source of the multicast stream and spanning the MCS's relevant to the stream destinations. This paper considers two heuristics: MaxCap and KMB*. MaxCap selects the widest (in terms of available bandwidth) suitable and hop limited point-point routes between the source MCS and each destination MCS and combines them to form a loopless point-multipoint connection. $\mathrm{KMB}^{*}$ modifies the widely considered $\mathrm{KMB}$ algorithm [Kou, Markowsky, Berman, 1981], to introduce the hop limit and allow for application to directed graphs whose edge weights represent the available link capacity. The performance of MaxCap and $\mathrm{KMB}^{*}$ are compared by means of simulation on random graphs in a static communication scenario, that does not consider the possible addition/dropping of MCS's to/from a connection, resulting from the reconfiguration of the active calls.

While architectures for multicasting in ATM networks have not yet been proposed in the technical literature, several algorithms for multicasting has been discussed, mostly with reference to datagram networks [Deering, 1990; Bharat-Kumar, 1983; Chow, 1991; Ballardie, 1993; Kompella, 1993; Deering, 1994]. Moreover, a vast amount of literature on exact algorithms and heuristics is available to solve the "Steiner problem in graphs" [Winter, 1987], that approaches this problem from the point of view of graph theory. These solutions cannot be applied to ATM networks for two main reasons. First, resource reservation is not performed in the case of connectionless data transfer but is required by ATM; as a consequence, algorithms that work only on undirected graphs, whose link weight do not represent the bandwidth availability, are not suitable for ATM multicasting. The undirected graph assumption is so established that it has been applied also in papers, e.g. [Jiang, 1992], that identify the available bandwidth as the key metric to solve the problem. Second, multicasting in datagram networks is approached by considering point-point routing schemes, e.g. reverse path routing, that are commonly used for data networks but do not suit to ATM networks. These issues are taken into consideration for the definition of MaxCap and KMB*.

The section 2 describes the network architecture. The two heuristics are formalized in the section 3 . The models used for the simulation are described in the section 4 . The performance results are discussed in the section 5 and the conclusions are given in the section 6 .

\section{NETWORK ARCHITECTURE}

The MLN is a logical network of MCS's and VP's, with topology and coverage area depending on the desired grade of connectivity, on the offered traffic and on the supported communication services. The MCS's receive and process all the requests for multipoint connection establishment, rearrangement and abatement and translate them into the most appropriate route configuration, according to the multipoint stream specification and to the network status.

MCS's interact one another to obtain information about the status of the MLN and the location of other MCS's. This is part of the point-point routing scheme, that adopts a limit on the maximum number of hops on a route, a useful approach to simplify the processing and 
save bearer resources under the assumption that a low hop count in the logical network implies a low hop count in the physical network.

As shown in Figure 1, one point-multipoint ATM connection is established by using a two-tier architecture, that combines one unidirectional point-multipoint connection with several bidirectional point-point ones. The point-multipoint connection is rooted at the MCS relevant to the stream source and spans the MCS's relevant to its destinations; a point-point connection attaches one communicating party to the relevant MCS's, under the control of the Local Exchange that may consider either static (e.g. location based) or dynamic (e.g. load controlled) association criteria. The main advantage of this architecture is the reduction of signaling traffic with respect to a single-tier architecture: this one implies the rearrangement of a point-multipoint connection each time an user adds to or drops from it, whereas the twotier architecture requires this each time an MCS adds to or drops from a connection.
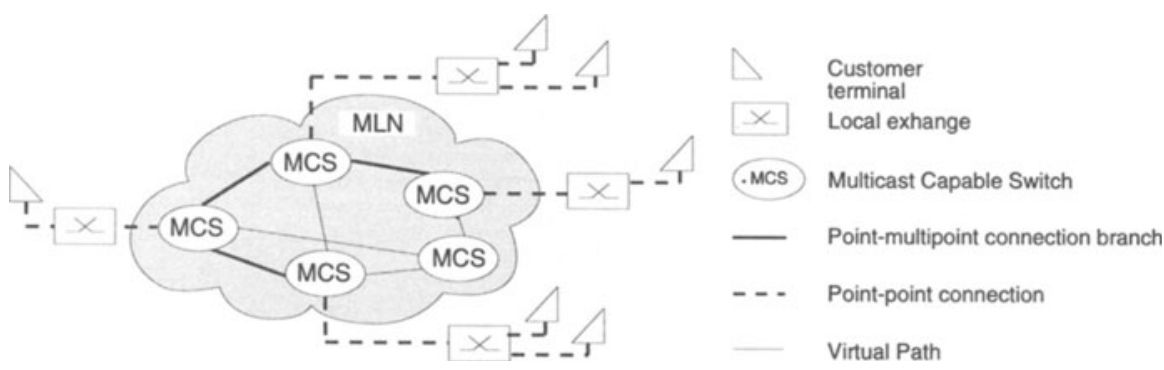

Figure 1 Multicast Logical Network Architecture.

The architecture of a MCS is sketched in Figure 2, for the case a Replication Server enhances a basic ATM Local Exchange with the capabilities required by multicasting. The Replication Server is composed of a number of entities, among which Figure 2 shows: a copy module in the User Plane to replicate the ATM cells, modules in the Control Plane to implement the [ITU-T, 1994; ITU-T, 1995] point-multipoint signaling procedures and the Multipoint Connection Controller, to collect the available arrangements to establish or modify a point-multipoint connection and select the best one.

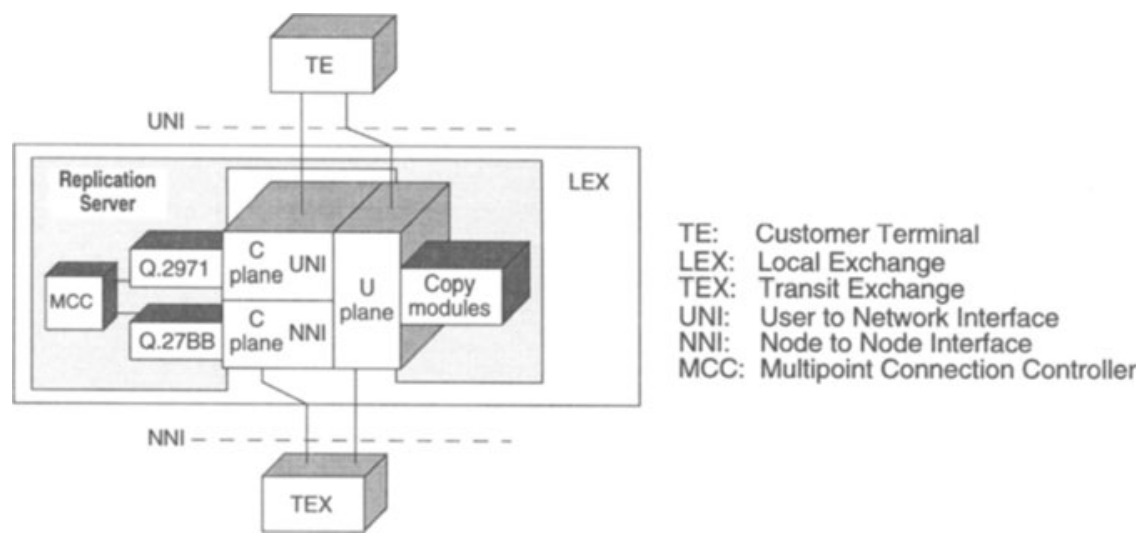

Figure 2 Architecture of a Multicast Capable Switch implemented as a server. 


\section{HEURISTICS FOR POINT-MULTIPOINT ROUTING}

The overlaid logical network of MCS's may be represented by a directed labeled graph $G=(V, E, C)$, wherein $V$ is the set of vertices associated to the MCS's, $E$ is the set of edges associated to the VP's between the MCS's and $C$ is a function that labels the edge $\left(v_{i}, v_{j}\right)$ with its available capacity $C_{i j}$. With reference to this graph, we formulate two heuristics for hop-limited, point-multipoint routing in directed graphs: $\mathrm{KMB}^{*}$ and MaxCap. These heuristics are parametric to maximum number of hops $(H)$ and aim at determining a loop-free point-multipoint route, rooted at a vertex $v_{0}$ (the MCS relevant to the stream source) and spanning a set of vertices $S \subseteq V$ (the MCS's relevant to the stream destinations).

$\mathrm{KMB}^{*}$ modifies the $\mathrm{KMB}$ algorithm [Kou, Markowsky, Berman; 1981] to take into account the value of $H$ and the link orientation. An important difference between KMB and $\mathrm{KMB}^{*}$ is that the former does not make any distinction between the vertices in $S$, as all of them play the role of both sender and receiver, whereas the latter clearly separates the source of a stream from the set $S$ of its destinations. $\mathrm{KMB}^{*}$ operates according to the following steps. 1. A complete directed labeled graph $G^{\prime}=\left(V^{\prime}, E^{\prime}, C^{\prime}\right)$ is constructed from $G$ in such a way that $V^{\prime}=S \cup\left\{v_{0}\right\}$ and, for every edge $e_{i j} \in E^{\prime}$, the associated label $C^{\prime}{ }_{i j}$ is set equal to the available capacity of the widest path from $v_{i}$ to $v_{j}$ in $G$. If more than one widest paths exist, then the one with minimum number of hops is selected (the reader may note that this last aspect is new with respect to the original KMB and allows for a lower cost in terms of consumed bandwidth). $G^{\prime}$ is constructed as follows:

1.1. let: $V^{\prime}=S \cup\left\{v_{0}\right\} ; E^{\prime}=\left\{e_{i j}=\left(v_{i}, v_{j}\right) \mid v_{i}, v_{\mathrm{j}} \in V^{\prime}\right) ; C^{\prime}{ }_{i j}=0$ for all the pairs $\left(v_{i}, v_{j}\right)$

1.2. for each vertex $v_{k} \in V^{\prime}$

1.2.1. $B=V^{\prime}-\left\{v_{k}\right\}$ is the set that holds the non visited nodes; each vertex $v_{i} \in V^{\prime}$ is associated a couple $\left(H_{k i}, D_{k i}\right)$, where $H_{k i}$ and $D_{k i}$ are the number of hops and the available capacity of the widest path from $v_{k}$ to $v_{i}$, respectively. $\left(H_{k i}, D_{k i}\right)$ is initialized at

$$
\left(H_{k i}, D_{k i}\right)= \begin{cases}(0, \infty) & \text { if } i=k \\ \left(1, C_{k i}\right) & \text { if } i \neq k \text { and } e_{k i} \text { exists } \\ (\infty, 0) & \text { otherwise }\end{cases}
$$

1.2.2. while the set $V^{\prime} \cap B$ is not empty

1.2.2.1. find the index $j$ such that $D_{k j}=\max \left\{D_{k i}\right\}$; if more than one vertices $v_{i} \in B$

$v_{i}$ have the same maximum value of $D_{k j}$ then select at random one among those with minimum $H_{k i}$; let $C^{\prime}{ }_{k j}=D_{k j}$.

1.2.2.2. let $B=B-\left\{v_{j}\right\}$.

1.2.2.3. each couple $\left(H_{k i}, D_{k}\right)$ of $v_{i} \in B$ is updated if $H_{k j}<H$ and the edge $e_{j i}$ exists; the new value is:

$$
\left(H_{k i}, D_{k i}\right)= \begin{cases}\left(H_{k j}+1, \min \left(D_{k j}, C_{j i}\right)\right) \text { if } \min \left(D_{k i}, C_{j i}\right)>D_{k i} \\ \left(H_{k j}+1, D_{k j}\right) & \text { if } \min \left(D_{k i}, C_{j i}\right)=D_{k i} \text { and } H_{k j}+1<H_{k i} \\ \left(H_{k j}, D_{k j}\right) & \text { otherwise }\end{cases}
$$

2. Find the spanning tree $T_{2}=\left(V_{2}, E_{2}\right)$ of $G^{\prime}$ with widest path. When inserting a vertex in $T_{2}$, only the edges that respect the limit on the number of hops are considered among those that have maximum capacity. Formally, each edge $e_{k i}$ of the complete graph $G$ ' is associated to the couple $\left(H_{k i}, D_{k i}\right)$, associated to the vertex $v_{i}$ in the step 1.2. when $v_{k}$ is considered. Moreover, each vertex $v_{j}$ is given a value $h_{j}$, representing the number of hops 
necessary to reach the vertex $v_{j}$ from the vertex $v_{0}$ following the path imposed by $T_{2}$. The procedure to build $T_{2}$ is as follows:

2.1. Let $V_{2}=\left\{v_{0}\right\}, E_{2}=\{\}, h_{0}=0$.

2.2. While the set $V^{\prime}-V_{2}$ is not empty:

2.2.1. define the set $E_{3}=\left\{e_{i j}=\left(v_{i}, v_{j}\right) \mid v_{i} \in V_{2}, v_{\mathrm{j}} \in V^{\prime}-V_{2}, h_{\mathrm{i}}+H_{i j}<=H\right\}$

2.2.2. select the edge $e_{i j} \in E_{3}$ with maximum $D_{i j}$; if more than one edges with maximum capacity exist, then select at random one among those with minimum $H_{i j}$.

2.2.3. let $V_{2}=V_{2}+\left\{v_{j}\right\} ; E_{2}=E_{2}+\left\{e_{i j}\right\} ; h_{j}=h_{i}+H_{i j}$

3. Construct the subgraph $G_{S}$ of $G$ by replacing each edge in $T_{2}$ by its corresponding path in $G$. For this purpose, one edge each in $T_{2}$ is considered at a time and, if the corresponding path leads to a vertex already reached by another path in $G$, then all the vertices and edges belonging to the longest path are eliminated. This may occur since intermediate vertices on a path in $G$ may belong to the set $S$ and is to be avoided for $T_{2}$ to be a tree. In order to remove loops we have chosen to eliminate the longest paths.

MaxCap implements a modified, hop limited, Dijkstra based routing that considers the link capacity to select the branches of a point-multipoint connection. For this purpose, it relies on the routing scheme proposed in [LeBoudec, Przygienda, Sultan, 1994], that incorporates a "widest path metric" into the well known Djikstra shortest path routing scheme. A similar approach is also used in [Shacham, 1992], to compute the maximum bandwidth shortest path for a point-multipoint connection, but it does not consider the hop limit and the directed graph model. The point-multipoint connection is built by assembling, loop-free, a set of point-point routes, each one meeting the constraint on the maximum number of hops.

MaxCap mainly differs from $\mathrm{KMB}^{*}$ for a modification of the step 1 and the lack of step 2. The step 1 is modified so that, in the step 1.2 , only $v_{k}=v_{0}$ is considered instead of taking into account all the vertices $v_{k} \in V^{\prime}$. Thus, MaxCap simplifies KMB* by selecting, at the end of step 1., the tree rooted at $v_{0}$ and composed of all the edges outgoing from the root and relevant to the vertices in $S$. It results that MaxCap selects the minimum length widest available path, whereas KMB* selects the widest paths so as to reduce the global cost in terms of consumed capacity, that is obtained by sharing as much as possible the links to the destinations.

The algorithmic complexity is an important index of performance of tree-building heuristics. From the above description it comes out that the complexity of MaxCap is lesser than that of $\mathrm{KMB}^{*}$ for a factor $|V|$. In fact, the complexity of $\mathrm{KMB}^{*}$ is $|S||V|^{2}$, because its three steps have complexity of the order of $|S||V|^{2},|S|^{3}$ and $|S||V|$ and $|S| \leq|V|$, and the resulting complexity of MaxCap is $|S||V|$, that is the complexity of each of its steps.

\section{PERFORMANCE METRICS AND SYSTEM MODEL}

The performance of MaxCap and $\mathrm{KMB}^{*}$ are evaluated by means of simulation on random graphs. Taking advantage of the adopted two tier multicasting architecture, these graphs need to represent only the MLN topology and the connections between the customers and the MCSs', disregarding the other switches and links. The following indices of performance are considered to evaluate the performance of MaxCap and $\mathrm{KMB}^{*}$ :

- $P$ : percentage of the communicating parties that are not attached to one point-multipoint connection;

- $S$ : number of stream copies made by the source and transit MCS's for each point-multipoint connection;

- $C_{S}$ : number of consecutive connections set-up with $P=0$.

$P$ measures the party blocking probability in a point-multipoint connection; $S$ gives an idea 
on the processing and traffic load on the MCS's; $C_{S}$ measures the number of multicast connections that may be concurrently accommodated by the considered heuristics.

To evaluate the above parameters for a number of different MLN mesh topologies, the random graph model proposed in [Waxman, 1988] has been modified to generate fully meshed networks and to make the edge probability independent of its length. The edge probability function is $P_{u, v}=G \exp \{[-d(u, v) * R] /[D-d(u, v)]\}$, wherein $d(u, v)$ is the Euclidean distance between the MCS's $u$ and $v, D$ is the maximum possible distance between any two MCS's, $R$ and $G$ are defined in $[0,1]$ and give the ratio of short to long edges and the grade of connectivity of the MLN, respectively. The values of $R$ and $G$ considered for the performance evaluation are shown in Table 1.

Table 1 Random mesh MLN's

\begin{tabular}{lllllll}
\hline & mesh \#1 & mesh \#2 & mesh \#3 & mesh \#4 & mesh \#5 & mesh \#6 \\
\hline $\mathrm{G}$ & 1 & 1 & 1 & 0.5 & 0.5 & 0.5 \\
$\mathrm{R}$ & 0 & 0.5 & 1 & 0 & 0.5 & 1 \\
\hline
\end{tabular}

The $N$ MCS's in a MLN are randomly located in the $(\mathrm{x}, \mathrm{y})$ plane. The edges are not weighted, to represent a MLN made of homogeneous MCS's that present the same level of load and usage cost. The links are weighted by the available capacity of the relevant VP, drawn from the probability distributions shown in Table 2 (the values are expressed in stream units, the maximum VP capacity is assumed equal to 10 stream units).

Table 2 Link capacity probability distribution

\begin{tabular}{lllll}
\hline Link capacity & D1 & D2 & D3 & D4 \\
\hline 1 & 0.3 & 0.1 & 0.3 & 0 \\
3 & 0.4 & 0.6 & 0.6 & 1 \\
10 & 0.3 & 0.3 & 0.1 & 0 \\
\hline
\end{tabular}

Each multicast group has a constant number $M$ of members. Two different multicast groups are considered: one is related to a multiparty conference communication, where each member transmits to and receives from all the other members ( $M$ stream sources per group and $M-1$ destinations per stream); the other group is related to a distributive communication from a single source to $M-1$ destinations. A multicast group is created by randomly generating $M$ pairs of $\{$ MCS number, party number . The members of a group are attached to different MCS's. Information streams are modeled as constant bit rate flows with unitary capacity.

No connectivity check is carried out after the generation of a MLN and a multicast group and it may occurs that some members of a group are not reachable from the others. That models the possible network inability in accommodating all the parties in a call, as a result of the bandwidth previously allocated to other in progress communications. The percentage of unreachable destinations $\left(P_{D U}\right)$, measured over 1500 pairs \{random network, random group \} for $M=5$, is shown in Table 3 for the six considered MLN topologies. The values of $P_{D U}$ are independent of the multicasting heuristic and of the link capacity, as they are evaluated for each stream as if it were the first one to be allocated for the group.

Table $3 P_{D U}$ values for the six considered MLN's

\begin{tabular}{lllllll}
\hline & mesh \#1 & mesh \#2 & mesh \#3 & mesh \#4 & mesh \#5 & mesh \#6 \\
\hline$P_{D U}$ & 0.0 & 0.0017 & 0.0442 & 0.0487 & 0.2202 & 0.4013 \\
\hline
\end{tabular}

\section{PERFORMANCE RESULTS}

To evaluate the performance of the two multicast heuristics, 1500 trials are carried out for each combination of the values of $G, R, N, M$ and $H$ and for two different communication services: videoconferencing and TV distribution. 
As for videoconferencing, MaxCap and $\mathrm{KMB}^{*}$ are applied at each trial to one pair of random MLN and multicast group. The $M$ streams are sequentially considered according to their order of generation and every member of the multicast group becomes, in turn, the root of a point-multipoint connection; as a consequence, the $i$ th stream in a sequence finds the network status modified by the previous $i-I$ streams $(2 \leq i \leq M)$. The outcome of each stream allocation by each heuristic is stored for post processing, so as to obtain the mean value of $P$ and $S$ for the $i$ th stream $(1 \leq i \leq M)$.

Tables 4 to 8 compare the $P$ performance of $\mathrm{KMB}^{*}$ and MaxCap for $N=10, M=5$ and $H=2$, varying the link capacity distribution. $P$ is always equal to zero for the mesh number 1 and the relevant valus are not shown in the Tables 5 to 8. The tables show that:

- $\mathrm{KMB}^{*}$ and MaxCap have the same performance for the stream number 1 (see Table 4) and this is the value of $P_{D U}$ reported in Table 3; in fact the step 1 of the two heuristics ensures that a solution is found whenever it exists; this solution is related to the mesh connectivity and is independent of the amount of available capacity;

- for the streams 2 to 5 a slight difference is appreciated in favor of KMB* only in a limited number of cases (shown unshaded in the Tables 5 to 8 ); that means that $\mathrm{KMB}^{*}$ is more able than MaxCap to avoid the utilization of links with very low capacity and to aim at an overall equalization of the capacity of the links in an MLN;

- $P$ is higher for D1 and D3, that have highest probability of unitary bandwidth links;

- the MLN connectivity degree affects heavily the $P$ values: the full mesh MLN (mesh 1) accommodates always all the streams in a multicast group, whereas the mean value of $P$ approaches $1 \mathrm{E}-2$ in the mesh 4 and is higher than $4 \mathrm{E}-1$ in the mesh 6;

- the last streams in a group suffer for a high mean value of $P$, for the reduced bandwidth in the MLN; this is more evident in the highly connected topologies, where the stream 5 has $P$ values that are even 3 - 4 times larger that those of the stream 1 ; that is less evident in the case of meshes with low connectivity because $P$ is high even for the stream 1;

- there is no difference between $\mathrm{KMB}^{*}$ and MaxCap if D4 is applied; consequently, the Table 8 refers only to MaxCap.

Table $4 \mathrm{KMB}^{*}$, MaxCap: $P$ performance for the stream number $1(N=10, H=2$, any D)

\begin{tabular}{lllrll}
\hline Mesh \# 1 & Mesh \# 2 & Mesh \# 3 & Mesh \# 4 & Mesh \# 5 & Mesh \#6 \\
\hline 0.0 & 0.0017 & 0.0442 & 0.0487 & 0.2202 & 0.4013 \\
\hline
\end{tabular}

Table $5 \mathrm{KMB}^{*}$ vs. MaxCap: $P$ performance $(N=10, \mathrm{D} 1, H=2)$

\begin{tabular}{llllllllll}
\hline Mesh \# & KMB & & \multicolumn{7}{c}{ MaxCap } \\
& stream 2 & stream 3 & stream 4 & stream 5 & stream 2 & stream 3 & stream 4 & stream 5 \\
\hline 2 & 0.0027 & 0.0032 & 0.0033 & 0.0065 & 0.0027 & 0.0032 & 0.0033 & 0.0065 \\
3 & 0.0517 & 0.0540 & 0.0573 & 0.0672 & 0.0518 & 0.0540 & 0.0573 & 0.0673 \\
4 & 0.0580 & 0.0650 & 0.0752 & 0.0832 & 0.0580 & 0.0653 & 0.0755 & 0.0837 \\
5 & 0.2388 & 0.2497 & 0.2713 & 0.2872 & 0.2390 & 0.2497 & 0.2718 & 0.2877 \\
6 & 0.4228 & 0.4357 & 0.4475 & 0.4782 & 0.4228 & 0.4357 & 0.4477 & 0.4785 \\
\hline
\end{tabular}

Table $6 \mathrm{KMB}^{*}$ vs. MaxCap: $P$ performance $(N=10, \mathrm{D} 2, H=2)$

\begin{tabular}{llllllllll}
\hline Mesh \# & \multicolumn{1}{c}{ KMB* } & \multicolumn{7}{c}{ MaxCap } \\
& stream 2 & stream 3 & stream 4 & stream 5 & stream 2 & stream 3 & stream 4 & stream 5 \\
\hline 2 & 0.0027 & 0.0030 & 0.0035 & 0.0043 & 0.0027 & 0.0030 & 0.0035 & 0.0043 \\
3 & 0.0449 & 0.0460 & 0.0487 & 0.0510 & 0.0449 & 0.0465 & 0.0488 & 0.0512 \\
4 & 0.0518 & 0.0530 & 0.0575 & 0.0598 & 0.0518 & 0.0530 & 0.0575 & 0.0598 \\
5 & 0.2210 & 0.2215 & 0.2283 & 0.2372 & 0.2210 & 0.2215 & 0.2283 & 0.2372 \\
6 & 0.4085 & 0.4095 & 0.4127 & 0.4227 & 0.4085 & 0.4095 & 0.4127 & 0.4230 \\
\hline
\end{tabular}


Table $7 \mathrm{KMB}^{*}$ vs. MaxCap: $P$ performance $(N=10, \mathrm{D} 3, H=2)$

\begin{tabular}{lllllllll}
\hline Mesh \# & \multicolumn{1}{c}{ KMB* } & \multicolumn{7}{c}{ MaxCap } \\
& stream 2 & stream 3 & stream 4 & stream 5 & stream 2 & stream 3 stream 4 stream 5 \\
\hline 2 & 0.0032 & 0.0037 & 0.0043 & 0.0067 & 0.0032 & 0.0037 & 0.0043 & 0.0067 \\
3 & 0.0517 & 0.0540 & 0.0580 & 0.0685 & 0.0518 & 0.0540 & 0.0582 & 0.0687 \\
4 & 0.0580 & 0.0650 & 0.0752 & 0.0845 & 0.0580 & 0.0653 & 0.0755 & 0.0850 \\
5 & 0.2388 & 0.2497 & 0.2722 & 0.2892 & 0.2390 & 0.2497 & 0.2727 & 0.2893 \\
6 & 0.4228 & 0.4357 & 0.4485 & 0.4790 & 0.4228 & 0.4357 & 0.4487 & 0.4793 \\
\hline
\end{tabular}

Table 8 MaxCap: $P$ performance $(N=10, \mathrm{D} 4, H=2)$

\begin{tabular}{lllll}
\hline Mesh \# & stream 2 & stream 3 & stream 4 & stream5 \\
\hline 2 & 0.0025 & 0.0027 & 0.0030 & 0.0035 \\
3 & 0.0447 & 0.0447 & 0.0449 & 0.0452 \\
4 & 0.0503 & 0.0507 & 0.0510 & 0.0545 \\
5 & 0.2217 & 0.2221 & 0.2224 & 0.2230 \\
6 & 0.4020 & 0.4028 & 0.4032 & 0.4032 \\
\hline
\end{tabular}

Figures 3 to 6 show the influence of $H$ on the P performance of MaxCap; similar values are also obtained for $\mathrm{KMB}^{*}$. These figures show that.

- there is almost no benefit in increasing the value of $H$ when the MLN is highly connected; this is line with the present trends in the design of point-point routing mechanisms for signaling and multiservice intelligent networks, that take advantage of the network full connectivity to rely on a non-hierarchical two-hop limited scheme;

- increasing $H$ is a proper way to boost the performance when the mesh is poorly connected; the performance gain is around $10 \%$ for the mesh number 6 and the stream number 5 , when going from $H=2$ to $H=4$, and goes up to more than $100 \%$ for the mesh number 6 and the stream number 1 , from $H=2$ to $H=4$;

- medium connected MLN's with a few tens of MCS's do not present any advantage when going from $H=3$ to $H=4$; the improvement is remarkable from $H=2$ to $H=3$;

- D2 and D4 (that feature a lower probability of links with unitary capacity but do not have overall higher average bandwidth) have slightly lower values of $P$ and a reduced variability of $P$ with $H$ than D1 and D3.

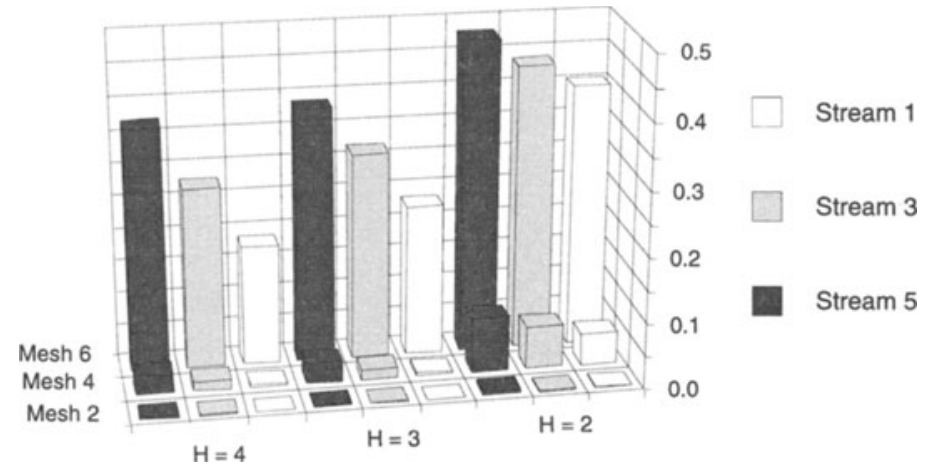

Figure $3 P$ performance of MaxCap for variable $H(N=10, M=5, \mathrm{D} 1)$. 


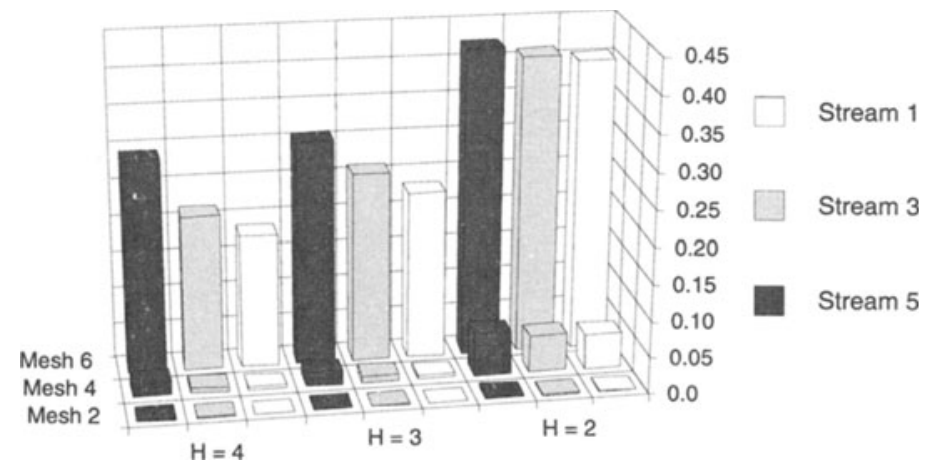

Figure $4 P$ performance of MaxCap for variable $H(N=10, M=5, \mathrm{D} 2)$.

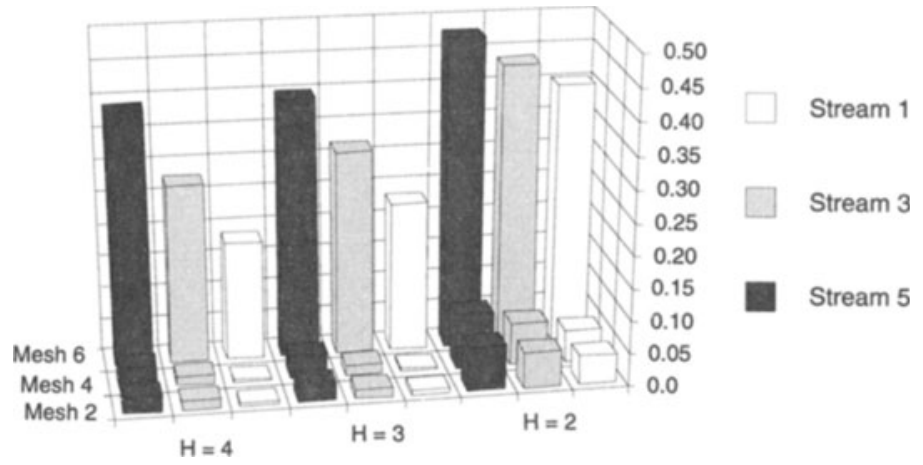

Figure $5 P$ performance of MaxCap for variable $H(N=10, M=5, \mathrm{D} 3)$.

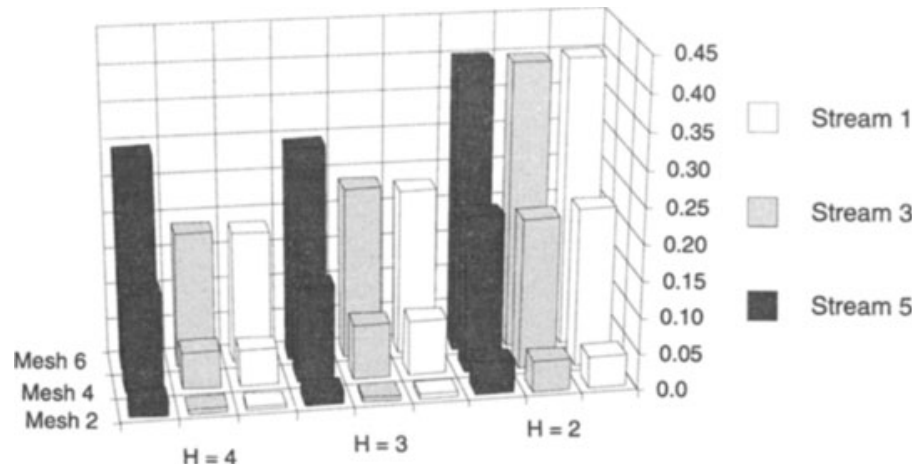

Figure $6 P$ performance of MaxCap for variable $\mathrm{H}(N=10, M=5$, D4). 
Table 9 compares the performance attained with $N=20$ and $M=5$ to those relevant to $N=10$ and $M=5$. The comparison is shown only for MaxCap, as these values are also representative of $\mathrm{KMB}^{*}$ performance under the same conditions. $P=0$ for every stream in the mesh 1 and 2 .

Table $9 P$ performance of MaxCap with variable $N(\mathrm{D} 2, H=2)$

\begin{tabular}{|c|c|c|c|c|c|c|c|c|c|c|}
\hline \multirow[t]{2}{*}{$\overline{\text { Mesh \# }}$} & \multirow{2}{*}{$\begin{array}{l}N=10 \\
\text { stream }\end{array}$} & \multicolumn{9}{|c|}{$N=20$} \\
\hline & & eam & rea & rea & 4 strea & 5 stre & stre & Zstr & 3 str & str \\
\hline 3 & 0.005 & 0.005 & 0.005 & 0.006 & 0.005 & 0 & 0 & 0 & 0 & 0 \\
\hline 4 & 0.003 & 0.003 & 0.002 & 0.002 & 0.003 & 0 & 0 & 0 & 0 & 0 \\
\hline 5 & 0.056 & 0.059 & 0.063 & 0.060 & 0.063 & 0 & 0.001 & 0.002 & 0.003 & 0.003 \\
\hline 6 & 0.195 & 0.198 & 0.194 & 0.195 & 0.204 & 0.024 & 0.024 & 0.027 & 0.029 & 0.041 \\
\hline
\end{tabular}

Figure 7 plots the mean value of $S$ for MaxCap and $\mathrm{KMB}^{*}$ for $N=10, M=5$ and D1. Again, $\mathrm{KMB}^{*}$ presents a slight advantage over MaxCap and the difference increases with $H$. Additional information may be obtained from Figure 8, that plots the mean number of copies made by the root of the point-multipoint connection in the scenario of Figure 7: KMB* builds slightly shorter paths with a higher degree of link sharing among the destinations of a group and involves less extra-MCS's in addition to those relevant to the group members.

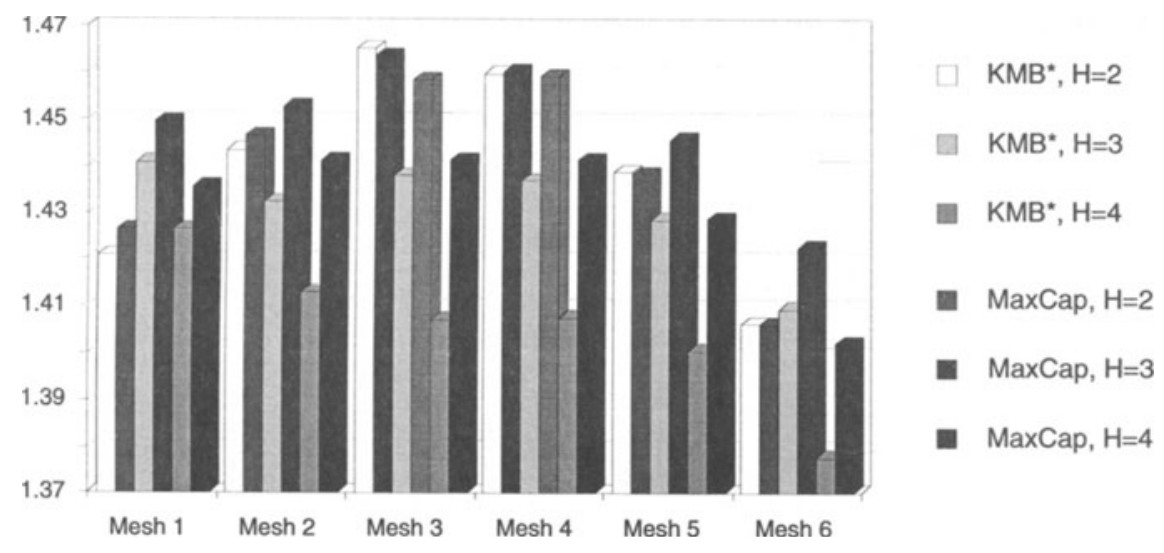

Figure 7 MaxCap vs. KMB*: $S$ performance for variable $H(N=10, M=5, \mathrm{D} 1)$.

In the case of distributive communication service, just one stream is generated for each multicast group and, consequently, one connection is to be established. A full mesh MLN, whose links have capacity equal to 10 , is generated to support a sequence of long-lived connections, each one obtained by randomly generating a multicast stream with $M-1$ destinations. The $i$ th multicast stream finds the network status modified by the previous $k$ streams $(1 \leq k<i)$ and, for each heuristic, the simulation terminates in correspondence of the first blocked stream $\left(i=C_{S}\right)$, i.e. the first time the heuristic fails in successfully covering the set of $M-1$ destinations of a multicast stream. Again, 1500 trials are repeated to obtain the distribution function of $C_{S}$. 


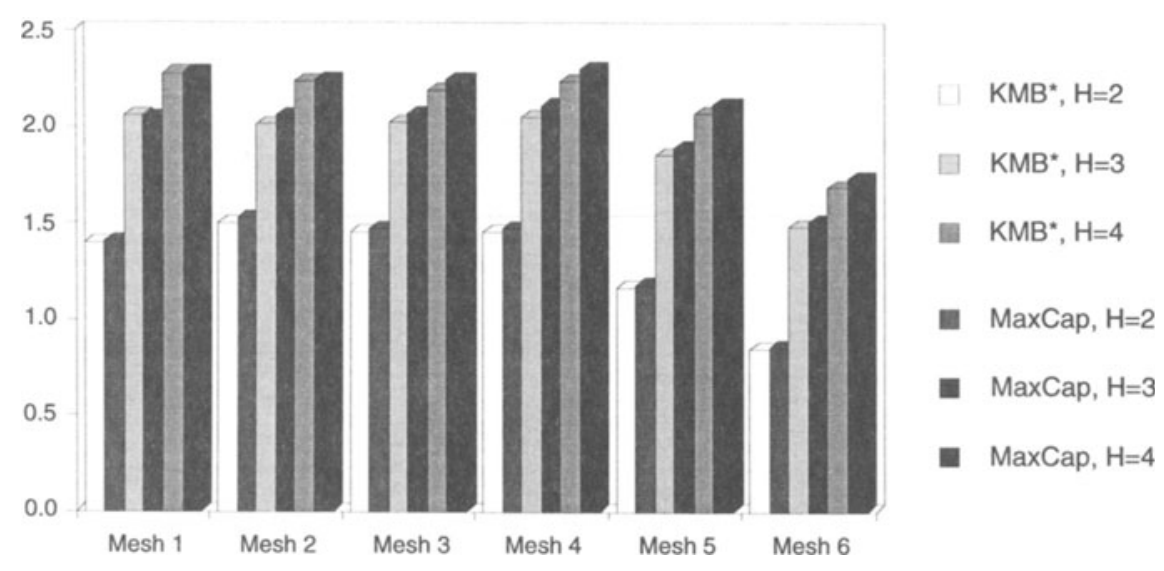

Figure 8 MaxCap vs. KMB*: average number of copies made by the root MCS for variable $H(N=10, M=5, \mathrm{D} 1)$

Figure 9 shows plots the distribution function of $C_{S}$, for a full mesh with $N=10$, initial link capacity equal to $10, M=5$ and $H=2,3$. As a consequence of its better utilization of the link capacity and shorter path length, $\mathrm{KMB}^{*}$ is able to accommodate more concurrent connections than that MaxCap; anyway, for a given probability, the difference between the number of established connections is, in any case, lower than $3 \%$.

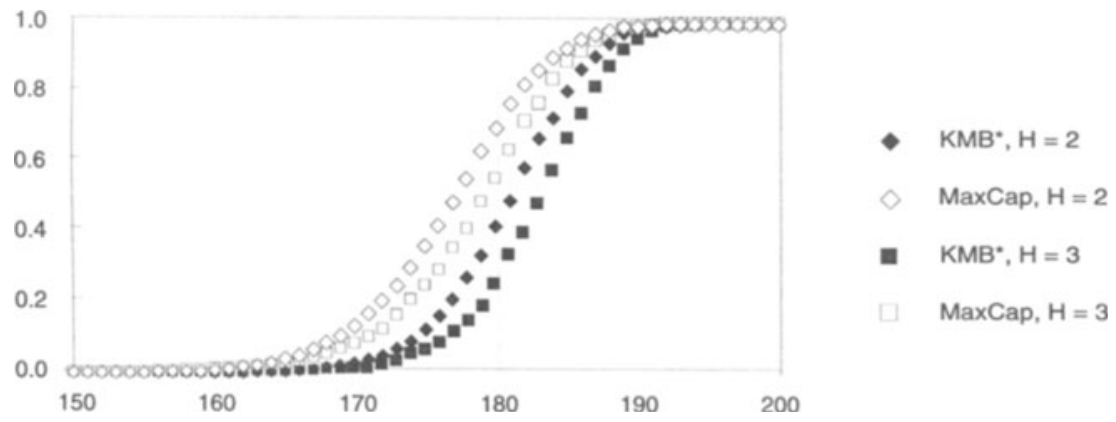

Figure 9 Distribution function of $C_{S}$ for MaxCap and KMB*, with $H=2,3($ Mesh 1, $M=5$ )

\section{CONCLUSIONS}

This paper proposes an architecture and two heuristics for multicasting in ATM networks, in presence of a constraint on the maximum number of hops on the routes that form the point-multipoint connection and modeling the considered network by means of a directed graph. One heuristic modifies the well known KMB algorithm for solving the Steiner tree problem in networks; the other one relies on a simple modification of the Dijkstra mechanism to take into account the link capacity in the construction of the connection. The performance evaluation shows that the two heuristics behave similarly under many different operating conditions, with just some slight advantage attained by the modified KMB algorithm; this has 
to be traded off against its higher algorithmic complexity. Further work is required to compare these heuristics in a dynamic environment, more suitable to represent the addition/dropping of users to/from an active connection. Moreover, additional analysis is required to evaluate alternative designs for the Multicast Logical Network topology and architecture (e.g. placement of the Multicast Capable Switches) and to understand the implications of the resulting logical network architecture on the physical network.

\section{ACKNOWLEDGEMENT}

Work carried out in the framework of the agreement between the Telecom Italia and the Fondazione Ugo Bordoni

\section{REFERENCES}

G.R.Ash, J.S.Chen, A.E.Frey, B.D.Huang: "Real Time Network Routing in a dynamic classof-service network", Proceedings of ITC 13, pp. 187-194

T.Ballardie, P.Francis, J.Crowcroft: "Core based tree"; ACM SIGCOMM '93; pp. 85-95

K.Bharat-Kumar, J.M.Jaffe: "Routing to multiple destinations in computer networks"; IEEE Transactions on communications; Vol. COM-31, No. 3, March 1983, pp. 343-351;

C.H.Chow: "On multicast path finding algorithms"; IEEE INFOCOM 1991, pp. 1274-1282

S.E.Deering, D.R.Cheriton: "Multicast routing in datagram internetworks and extended LAN's"; ACM Transaction on Computer Systems, Vol. 8, No.2, May 1990, Pages 85-110

S.Deering, D.Estrin, D.Farinacci, V.Jacobson, C.Liu, L.Wei: “An architecture for wide-area multicast routing"; Proceedings of ACM SIGCOMM '94; pp.126-135

ITU-T Draft Recommendation Q.2971: "Point to multipoint call/connection control"; Geneva Sept. 1994

ITU-T Draft Recommendation Q.27BB “Point-to-multipoint B-ISUP”; 1995

J.Y.LeBoudec, B.Przygienda, R.Sultan,: "Routing metric for connections with reserved bandwidth"; 12th EFOC\&N conference, June 1994, pp. 84-87

X.Jiang: "Routing broadband multicast streams"; Computer Communications, Vol. 15. No. 1, pp. $45-51$

V.P.Kompella, J.Pasquale, G.C.Polyzos: "Multicast routing for multimedia communication"; IEEE/ACM Transaction on networking, Vol. 1. No. 3, June 1993, pp. 286-292

L.Kou, G.Markowsky, L.Berman: “A fast algorithm for Steiner trees”; Acta Informatica, 15, $141-145,1981$

N.Shacham: "Multipoint communications by hierarchically encoded data"; IEEE Infocom '92; paper 9A.4

B.Waxman: "Routing of multipoint connections"; IEEE JSAC, Dec. 1988, pp.1617-1622

P.Winter: "Steiner Problems in Networks"; Networks, Vol. 17 (1987), pp. 129-167 